\title{
Vitrectomia transconjuntival 25 gauge via pars plana para opacidade vítrea persistente em pacientes com implante de lentes multifocais
}

\author{
Transconjunctival sutureless 25-gauge vitrectomy \\ for visually significant vitreous floaters in patients \\ with multifocal intraocular lens
}

Leonardo Akaishi' ${ }^{1}$, Fábio Canamary², Patrick Frensel de Moraes Tzelikis ${ }^{3}$

\begin{tabular}{|c|}
\hline ABStraCt \\
\hline $\begin{array}{l}\text { Purpose: The aim of this study was to evaluate the role of } 25 \text {-gauge transconjuntival } \\
\text { sutureless vitrectomy (TSV) in patients with persistent vitreous floaters who underwent } \\
\text { multifocal intraocular lens (IOL) implantation. Methods: Fourteen eyes of } 11 \text { patients } \\
\text { with multifocal IOL implant associated with vitreous opacities that underwent } 25 \text {-gauge } \\
\text { vitrectomy were prospectively analyzed. Information collected included near (UCNVA) } \\
\text { and distance uncorrected visual acuity (UCDVA), best corrected distance visual acuity } \\
\text { (BCDVA), and the spherical equivalent (SE) before and } 3 \text { months after vitrectomy. } \\
\text { Patients were questioned about surgery satisfaction and visual improvement after } \\
\text { vitrectomy. Results: Three months after } 25 \text {-gauge TSV the mean visual acuities were: } \\
\text { UCDVA, } 0,10 \pm 0,14 \text {; BCDVA, } 0,02 \pm 0,08 \text {; UCNVA, } 0,00 \pm 0,0 ; \mathrm{SE},+0,16 \pm 0,34 \text {. Thirteen } \\
\text { eyes }(92.9 \%) \text { had a UCDVA of } 0.5(20 / 40) \text { or better, and } 11(78.5 \%) \text { achieved a UCDVA } \\
\text { of } 0.8(20 / 25) \text { or better. Ten }(90.9 \%) \text { patients demonstrated satisfaction with surgery, } \\
\text { and floaters have gone away in thirteen eyes }(92.8 \%) \text {, while one patient expressed no } \\
\text { satisfaction with her postoperative visual acuity because of the cystoid macular edema } \\
\text { that occurred. Conclusion: } 25 \text {-gauge TSV was a safe and accurate option for patients } \\
\text { with multifocal lens and visual disturbing vitreous floaters providing subjective } \\
\text { improvement of symptoms. }\end{array}$ \\
\hline
\end{tabular}

Keywords: Vitrectomy; Lens implantation, Intraocular; visual acuity; Cataract extraction; Patient satisfaction; Treatment outcome

\footnotetext{
${ }^{1}$ Mestre, Chefe do Departamento de Catarata do Hospital Oftalmológico de Brasília - HOB - Brasília (DF), Brasil; ${ }^{2}$ Estagiário do Departamento de Catarata do Hospital Oftalmológico de Brasília - HOB - Brasília (DF), Brasil;

${ }^{3}$ Doutor, Médico Assistente do Departamento de Catarata do Hospital Oftalmológico de Brasília HOB - Brasília (DF), Brasil.

Trabalho realizado no Hospital Oftalmológico de Brasília - HOB - Brasília (DF), Brasil.

Os autores não apresentam nenhum interesse financeiro no material apresentado

Recebido para publicação em: 23/3/2010 - Aceito para publicação em 19/6/2010
}

Rev Bras Oftalmol. 2010; 69 (4): 252-8 


\section{INTRODUÇÃO}

A s opacidades vítreas (floaters) são bastante conhecidas como distúrbios visuais que podem ser acompanhadas objetivamente e subjetivamente de uma diminuição na acuidade visual. Embora geralmente sem nenhuma consequência, os floaters correspondem a alterações na arquitetura vítrea que geralmente resulta de um descolamento do vítreo posterior (DVP). O DVP consiste na liquefação do corpo vítreo e o enfraquecimento da aderência entre a cortical posterior do vítreo e a membrana limitante interna que posteriormente se separam. Com a idade o vítreo tornase mais fluido, de modo que sua movimentação aumenta muito com os movimentos do globo ocular. Apesar de menos comum, a presença de floaters sintomáticos pode resultar de rupturas ou descolamento de retina, hemorragia vítrea, inflamação intraocular, ou hialite asteróide. (1-3) Em uma pequena porcentagem de pacientes, os sintomas visuais relacionados aos floaters podem ser significativos. Para estes pacientes, um tratamento mais agressivo pode ser necessário na tentativa de reduzir o desconforto ocular produzido por essas opacidades vítreas.

As medidas de acuidade visual nem sempre refletem de maneira acurada o desconforto visual dos pacientes, e os sintomas oculares relacionados às opacidades vítreas podem se mostrar bastante debilitantes. Já foi demonstrado o papel da vitrectomia em pacientes com distúrbio visual significativo relacionado à presença de floaters, com a maioria dos pacientes relatando uma elevada satisfação com sua visão no período pós-operatório. ${ }^{(4-7)}$ Recentemente, com o advento de novos materiais com um novo sistema de vitrectomia transconjuntival permite hoje a realização de vitrectomia via pars plana sem sutura 25-gauge (VVPP-25-gauge). Tratase de uma técnica potencialmente menos invasiva e que não compromete a eficácia do procedimento. Além disso, apresenta um número variado de benefícios, como redução do tempo operatório, redução do desconforto do paciente, redução do processo inflamatório pós-operatório, reabilitação visual mais rápida, e menor risco teórico de irregularidade corneana no pós-operatório.

O objetivo do presente estudo é relatar o resultado visual obtido após realização de VVPP-25-gauge em pacientes com opacidades vítreas persistentes e debilitantes previamente submetidos à cirurgia de facectomia com implante de lente intraocular (LIO) multifocal.

\section{Métodos}

Este estudo clínico, prospectivo, intervencionista, foi desenvolvido no Setor de Catarata e Setor de Retina do Hospital Oftalmológico de Brasília - HOB, após ter sido analisado o protocolo e aprovado pelo Comitê de Ética Médica do HOB. Todos os pacientes incluídos neste estudo foram informados, de forma detalhada, sobre a natureza da investigação, técnica cirúrgica, possíveis riscos e complicações e alternativas de tratamento. Antes da cirurgia e após os devidos esclarecimentos, todos os pacientes assinaram o Termo de Consentimento incluído na pesquisa.

Foram analisados prospectivamente 14 olhos de 11 pacientes, tratados com VVPP-25-gauge, no período de agosto de 2006 a novembro de 2008. Foram incluídos pacientes com implante de LIO multifocal e que apresentavam opacidades vítreas persistentes há pelo menos 3 meses e intensamente desconfortáveis. Os critérios de exclusão foram: olho único, alteração sistêmica que pudesse alterar a cicatrização pós-operatória (diabetes mellitus, doenças autoimunes, doenças do tecido conjuntivo), enfermidade ocular pré-existente que pudesse alterar a acuidade visual ou contraindicar a cirurgia (doença ocular herpética, olho seco moderado ou grave, uveíte, glaucoma, doenças retinianas), e falta de colaboração para efetuar exames e a cirurgia.

\section{Técnica operatória de vitrectomia via pars plana 25-gauge}

Todas as cirurgias foram realizadas pelo mesmo cirurgião. No pré-operatório os pacientes utilizaram colírio antibiótico de quinolona de $4^{\mathrm{a}}$ geração gatifloxacina (Zymar ${ }^{\circledR}$, Allergan,EUA) ou moxifloxacina (Vigamox ${ }^{\circledR}$, Alcon, EUA) 4 vezes por dia (6/6h), dois dias antes da cirurgia. No dia da cirurgia, aproximadamente $30 \mathrm{~min}$ antes, iniciava-se a dilatação pupilar com solução de tropicamida a 1\% (Mydriacyl $®$,Alcon,EUA) e cloridrato de fenilefrina a 10\% (Allergan, EUA), numa proporção de 1:1 (uma gota de 5 em 5 min, por 3 vezes). A anestesia em todos os casos foi tópica, com instilação de cloridrato de lidocaína a 2\%, geléia, (Astra Zeneca,Argentina) sem vasoconstrictor no fundo de saco conjuntival, iniciado 30 min antes da cirurgia, duas vezes, com intervalo de 10 min. Por via endovenosa foi utilizado $1,0 \mathrm{mg}$ de midazolam $5,0 \mathrm{mg} / \mathrm{ml}$ (Roche, Brasil). Após assepsia e antissepsia da pele com iodo-povidona a $10 \%$ (Cinord Sul, Brasil), equivalente a $1 \%$ de Iodo ativo, um campo plástico adesivo era colocado em contato com as pálpebras dos pacientes, de forma a isolar os cílios do campo operatório, e o 
blefarostato inserido. Uma solução de iodo-povidona a 3\% (Ophthalmos, São Paulo) foi usada com finalidade anti-séptica no fundo de saco conjuntival após anestesia tópica.

Na cirurgia, com sistema VVPP-25-gauge, não era realizada peritomia conjuntival, sendo apenas necessário deslocar a conjuntiva para provocar um desalinhamento entre a conjuntiva e a incisão escleral. A primeira microcânula era introduzida diretamente através da conjuntiva $(3,5 \mathrm{~mm}$ posterior ao limbo) na região temporal-inferior na qual era introduzida a cânula de infusão. As outras duas microcânulas eram introduzidas nos quadrantes supero temporal e supero nasal. Uma vitrectomia completa e cuidadosa era então realizada com aparelho da Accurus ${ }^{\circledR}$ Surgical System (Alcon Surgical Laboratories, Fort Worth, Texas, EUA) com baixos parâmetros de aspiração e níveis elevados de corte, no intuito de evitar trações periféricas. Após remoção vítrea, era realizada uma identação com o intuito de avaliar a ausência de roturas periféricas, e para remover resquícios de vítreo periférico. Ao término da cirurgia as microcânulas eram removidas e não era feito sutura escleral uma vez identificado ausência de extravasamento de fluido.

No período pós-operatório, o paciente foi orientado para utilizar topicamente: cetorolaco de trometamina a 0,5\%, Acular $^{\circledR}$, Allergan Produtos Farmacêuticos, São Paulo, Brasil) quatro vezes ao dia por quatro semanas, gatifloxacina $\left(\right.$ Zymar $\left.^{\circledR}\right)$ ou moxifloxacina $\left(\right.$ Vigamox $\left.^{\circledR}\right)$ quatro vezes ao dia por 10 dias, e acetato de prednisolona a 1\% (Predfort ${ }^{\circledR}$, Allergan Produtos Farmacêuticos, São Paulo, Brasil) quatro vezes ao dia por quatro semanas. Medicação analgésica via oral também foi prescrita em caso de dor.Todos os pacientes foram avaliados no período pré-operatório e pós-operatório de 1 dia, 1 , e 3 meses.

\section{Pacientes examinados}

Os dados coletados incluem sexo, idade, comprimento axial, tempo entre a cirurgia de catarata e a cirurgia de VVPP-25-gauge, tipo de LIO multifocal implantada, acuidade visual sem correção (AVSC) pré e pósoperatória a vitrectomia, acuidade visual com correção (AVCC) para longe, acuidade visual para perto corrigida para longe (AVPC) a uma distância de $40 \mathrm{~cm}$, equivalente esférico (EE) pré e pós-operatório, e complicações. Neste estudo, as medidas de acuidade visual foram registradas em $\log$ MAR.

Um questionário relacionado à satisfação foi realizado ao final do estudo. A satisfação do paciente foi baseada em questões relacionadas à visão para longe, perto e direção noturna. A pontuação foi apresentada em uma escala que variava de 1 a 10 , sendo $1=$ incapacitante e $10=$ excelente. Os pacientes também foram questionados a respeito de fenômenos visuais (glare, halos) e orientados a responder de acordo com a seguinte escala de pontuação: $0=$ nenhum; 1 = mínimo; 2,3 , e $4=$ moderado $; 5=$ intenso.

A avaliação estatística dos resultados foi realizada através da utilização do programa estatístico SPSS para Windows, versão 12.0 Chicago: SPSS Inc; 2004. Foi realizado test t para comparação dos resultados pré-operatórios com pós-operatórios. As diferenças foram consideradas significativas quando $\mathrm{P}<0,01$.

\section{$\underline{\text { Resultados }}$}

Foram avaliados prospectivamente 14 olhos de 11 pacientes, destes 4 (36,4\%) eram homens e $7(63,6 \%)$ mulheres, com média de idade de 69,5 anos $\pm 5,2$ (desvio padrão - DP) (variando de 45 a 84 anos). A média de tempo entre a cirurgia de catarata e a VVPP-25-gauge foi de 5,5 \pm 6.7 meses (variando de 3 a 25 meses). $\mathrm{O}$ comprimento axial médio foi de $23,43 \mathrm{~mm} \pm 0,80 \mathrm{~mm}$ (variando de 22,29 a 25,79 mm). As opacidades vítreas foram consequência de hialose asteróide em 1 olho e descolamento do vítreo posterior em 13 olhos. Dos 14 olhos estudados, 12 olhos $(85,7 \%)$ receberam a LIO Tecnis ZM900 multifocal e dois olhos $(14,3 \%)$ a LIO ReSTOR multifocal.

Após cirurgia de catarata e antes da vitrectomia 25 -gauge, o EE médio foi $+0,07 \pm 0,45 \mathrm{D}$ (variando $-0,50$ a $+0,75 \mathrm{D})$. A AVSC média para longe após cirurgia de catarata foi de $0,12 \pm 0,08$ (variando 0,00 a 0,30 ), e a AVCC média para longe foi de $0,02 \pm 0,04$ (variando $0,00$ a 0,10$)$. Quatorze olhos $(100,0 \%)$ apresentavam AVSC para longe de $0,5(20 / 40)$ ou melhor, e $8(57,1 \%)$ atingiram AVSC de 0,8 (20/25) ou melhor.

Três meses após vitrectomia 25-gauge, o EE médio foi de $+0,16 \pm 0,34 \mathrm{D}$ (variando $-0,25 \mathrm{a}+0,50 \mathrm{D}$ ). A AVSC média para longe foi de $0,10 \pm 0,14$ (range 0,00 a 0,54 ), e a AVCC média para longe de $0,02 \pm 0,08$ (variando 0,00 a 0,30$)$. Treze olhos ( $92,9 \%$ ) obtiveram AVSC para longe de 0,5 (20/40) ou melhor, e $11(78,6 \%)$ de 0,8 (20/25) ou melhor. Diferenças no EE, AVSC e AVCC para longe antes e após vitrectomia 25-gauge não apresentaram diferença estatisticamente significativa ao final do último exame $(\mathrm{P}>0,05)$ (tabela 1$)$.

Não foram observadas complicações intraoperatórias e pós-operatórias como: hipotonia, extravasamento nas incisões, vítreo e retina encarcerado na ferida cirúrgica, descolamento de coróide, roturas 
Tabela 1

Resultado visual da acuidade visual para longe e perto ao final de 3 meses de seguimento pré e pós-vitrectomia

\begin{tabular}{lccc}
\hline Medidas & Pré-operatório & Pós-operatório & Valor de P \\
\hline AVSC (média) & $0,59 \pm 0,33$ & $0,10 \pm 0,14$ & 0,68 \\
AVSC de 20/25 ou melhor, n (\%) & $8(57,1 \%)$ & $11(78,6 \%)$ & - \\
AVSC de 20/40 ou melhor, n (\%) & $14(100 \%)$ & $13(92,9 \%)$ & - \\
AVPC (média) & $0,00 \pm 0,0$ & $0,00 \pm 0,02$ & 0,70 \\
AVPC de J1 ou melhor, n (\%) & $14(100 \%)$ & $13(92,9 \%)$ & - \\
AVCC (média) & $0,18 \pm 0.15$ & $0,00 \pm 0,01$ & 0,67 \\
\hline
\end{tabular}

AVSC: acuidade visual sem correção; AVPC: acuidade visual para perto corrigida para longe a uma distância de $40 \mathrm{~cm}$; AVCC: acuidade visual com correção para longe Medidas em logMAR

Tabela 2

Resultado do questionário relacionado à satisfação e a fenômenos visuais realizado 3 meses após a cirurgia de vitrectomia

\begin{tabular}{lccc}
\hline & \multicolumn{2}{c}{ Média de pontos \pm DP (Variação) } & \\
\cline { 2 - 2 } Questões & Pré-vitrectomia & Pós-vitrectomia & Valor de P \\
\hline $\begin{array}{l}\text { (*) Qual o grau de satisfação } \\
\text { com o resultado visual } \\
\text { obtido após a cirurgia? }\end{array}$ & $7,27 \pm 0,78(6$ a 8$)$ & $8,09 \pm 1,22(5,0$ a 9,0$)$ & $0,02 *$
\end{tabular}

Qual o grau de dificuldade em relação à seguinte tarefa:

$\begin{array}{lccc}\text { Visão para longe? } & 0,45 \pm 0,52(0 \text { a } 1) & 0,36 \pm 0,92(0 \text { a } 3) & 0,72 \\ \text { Visão para perto? } & 1,18 \pm 0,40(1 \text { a } 2) & 0,27 \pm 0,64(0 \text { a } 2) & 0,002 * \\ \text { Glare? } & 0,45 \pm 0,52(0 \text { a } 1) & 0,18 \pm 0,64(0 \text { a } 1) & 0,08 \\ \text { Visão noturna? } & 0,63 \pm 0,67(0 \text { a } 2) & 0,45 \pm 0,68(0 \text { a } 2) & 0,34 \\ \text { Halos? } & 1,00 \pm 0,63(0 \text { a } 2) & 0,72 \pm 0,64(0 \text { a } 2) & 0,08 \\ \text { Assistir TV? } & 0,45 \pm 0,52(0 \text { a } 1) & 0,18 \pm 0,60(0 \text { a } 2) & 0,19 \\ \text { Realizar leitura (jornal, revista) }) & 0,54 \pm 0,52(0 \text { a } 1) & 0,09 \pm 0,30(0 \text { a } 1) & 0,01 * \\ \text { Cozinhar? } & 0,36 \pm 0,50(0 \text { a } 1) & 0,18 \pm 0,60(0 \text { a } 2) & 0,34 \\ \text { Direção noturna? } & 0,81 \pm 0,60(0 \text { a } 2) & 0,63 \pm 0,67(0 \text { a } 2) & 0,34 \\ \text { Computador? } & 1,18 \pm 0,87(0 \text { a } 2) & 0,63 \pm 0,92(0 \text { a } 3) & 0,02 * \\ \text { Utilizar celular? } & 0,45 \pm 0,52(0 \text { a } 1) & 0,18 \pm 0,60(0 \text { a } 2) & 0,19 \\ \text { Barbear/Maquiagem? } & 1,09 \pm 1,1(0 \text { a } 3) & 0,81 \pm 0,98(0 \text { a } 3) & 0,08 \\ \text { Realizar compras? } & 0,54 \pm 0,52(0 \text { a } 1) & 0,27 \pm 0,64(0 \text { a } 2) & 0,19\end{array}$

(*)Escala de satisfação com pontuação que variava de 1 a 10 , sendo 1 = incapacitante e 10 = excelente.

Escala referente a todas as outras questões com a seguinte pontuação: $0=$ nenhum; $1=$ mínimo; 2, 3, e 4 = moderado; 5 = intenso.

retinianas, descolamento de retina e endoftalmite. A AVCC média para longe ao final de 3 meses de seguimento pós-vitrectomia 25-gauge não apresentou diferença quando comparado aos valores antes da cirurgia de vitrectomia $(\mathrm{P}=0,08)$. Três olhos $(21.4 \%)$ ganharam 1 linha de visão na AVCC para longe, 1 olho perdeu 1 linha de visão, e 1 olho perdeu duas linhas de visão porque desenvolveu edema macular cistóide, confirmada por angiografia fluorescente.

A queixa principal de todos pacientes foi a presença persistente e debilitante das opacidades vítreas que interferiam nas atividades diárias e no trabalho, especialmente para perto. A acuidade visual média antes da indicação da vitrectomia foi de 20/25 ou melhor, mas apesar deste nível de visão, todos os pacientes tinham embaçamento visual transitório causado pelas opacida- 
des que entravam no eixo visual. Os pacientes geralmente relatavam que necessitavam piscar ou mover a cabeça para que as opacidades se deslocassem para fora do eixo visual.

O questionário referente à satisfação dos pacientes mostrou que a grande parte dos pacientes estava satisfeita com o resultado visual obtido após vitrectomia 25-gauge (média de pontos $8,09 \pm 1,22$; variando de 5 a 9). Nenhum paciente relatou halos ou glare severos ou incapacitantes, sendo citado apenas como leve a moderado por alguns pacientes (Tabela 2). A maior parte dos pacientes $(90,9 \%)$ ficou satisfeita com o tratamento e afirmaram que sua ansiedade foi dramaticamente reduzida. As opacidades vítreas sumiram em 13 olhos ( $92,8 \%)$, com consequente melhora da qualidade funcional, enquanto apenas um paciente afirmou a não resolução das opacidades e demonstrou insatisfação com a acuidade visual pós-operatória, provavelmente pelo edema macular cistóide desenvolvido. Durante os 3 meses de seguimento nenhum paciente demonstrou recorrência subjetiva dos sintomas.

\section{$\underline{\text { Discussão }}$}

Mosca volante ou floaters é uma queixa comum dos pacientes que procuram consulta oftalmológica. Pode se apresentar como um sintoma indicativo de graves patologias vítreorretinianas, ou ocorrer comumente em olhos perfeitamente normais em decorrência a um descolamento de vítreo posterior (DVP), debris, degeneração filamentar e sinérese vítrea. ${ }^{(8-9)}$ Uma grande parte dos pacientes com floaters ao decorrer do tempo acaba por se habituar à presença dos mesmos, na qual a sua percepção no campo visual passa a ocorrer particularmente quando se observa uma superfície branca ou um espaço aberto monocromático. No entanto, em uma minoria dos casos, as opacidades vítreas podem tornarse persistentes, desconfortáveis e ocasionar redução na acuidade visual por obstruir o eixo visual. Nestes pacientes, o que se observa é um comprometimento na qualidade de vida.

A avaliação pré-operatória dos pacientes com moscas volantes deve incluir uma análise aprofundada do vítreo para determinar a presença e a posição das opacidades vítreas. Em 1983, Murakami et al. ${ }^{(10)}$ relatou alterações vítreas em 148 olhos com aparecimento súbito de moscas volantes. Eles observaram que $76 \%$ dos pacientes identificavam uma ou poucas moscas volantes e $24 \%$, identificavam um número elevado, sendo que as opacidades estavam localizadas principalmente na área central de visão. Oitenta e seis por cento dos pacientes tinham 50 anos ou mais de idade. Em nosso estudo, os pacientes tinham em média 65 anos ou mais, e o número de opacidades era geralmente descrito como reduzido por $72,7 \%$ dos pacientes. O descolamento do vítreo posterior (DVP) foi observado em $92,8 \%$ dos olhos após a cirurgia de catarata em nosso estudo. A detecção de um descolamento completo da face posterior do vítreo é essencial, porque um DVP induzido cirurgicamente pode causar rupturas da retina, aumentando o risco de descolamento de retina. ${ }^{(11)}$ Já é conhecido que a presença de floaters está geralmente relacionada à existência de um DVP. ${ }^{(10)}$ No presente estudo, dos 14 olhos com floaters, 12 apresentavam DVP.

Os sintomas apresentados pelos nossos pacientes incluíam visão borrada intermitente e opacidades móveis no campo visual, comprometendo principalmente atividades realizadas a pequena distância. No exame oftalmológico, apesar das queixas, todos os pacientes apresentavam uma excelente acuidade visual tanto para longe como para perto, o que evidencia que as medidas de visão realizadas através da tabela de Snellen não são suficientes para avaliar a qualidade visual destes pacientes. Como todos os pacientes eram pseudofácicos e submetidos a implante de lente intraocular multifocal, alterações como opacidade de cápsula posterior (OCP) e de superfície corneana (edema, olho seco) foram previamente descartadas como possíveis causas de comprometimento visual antes da indicação da vitrectomia. Além disso, os pacientes deveriam apresentar sintomas há pelo menos 3 meses.

No período pré e pós-vitrectomia, os pacientes foram submetidos a uma série de perguntas relacionadas à qualidade de visão para avaliar o ganho de visão após a realização da vitrectomia. O que o presente estudo pode identificar é que a grande maioria dos pacientes ficou satisfeita com a visão após o procedimento, sendo que o resultado foi ainda melhor em relação às atividades realizadas a uma pequena distância. Em relação à acuidade visual, medida pela tabela de Snellen, não foram observadas alterações significativas quando comparados o período pré e o pós-operatório da vitrectomia.

Entre as diferentes maneiras de se tratar a presença de floaters, a fotodisrupção com Nd:YAG laser já foi relatado com sucesso. ${ }^{(4)} \mathrm{Em} 1993$, Tsai et al. ${ }^{(4)}$ relatou quinze olhos submetidos ao procedimento de $\mathrm{Nd}$ :YAG laser para tratamento de floaters com muito sucesso e sem complicações, porém alguns pacientes relataram a presença contínua de opacidades menores sendo necessário, em alguns casos, a realização de várias sessões de laser. Este procedimento parece ser mais eficaz quando 
as opacidades são mais localizadas e menos difusas. Como desvantagem para o uso rotineiro de Nd:YAG laser no tratamento de alterações vítreas encontra-se o risco potencial de causar danos corio-retinianos como: hemorragia coroidal, hemorragia retiniana e danos ao epitélio pigmentar da retina. ${ }^{(12)}$ No entanto, estudos mostram que as complicações relacionadas ao uso de Nd:YAG laser estão relacionadas à potência do laser e a distância do foco do laser a retina. ${ }^{(13-14)}$

A vitrectomia via pars plana (VPP) com 20-gauge convencional também tem sido utilizada para remover floaters em pacientes selecionados. ${ }^{(5)}$ Várias vantagens da técnica de 25-gauge em relação a 20 gauge já foram descritos. Durante a realização da VPP 25-gauge certos passos desnecessários podem ser eliminados. Por exemplo, a necessidade de peritomia conjuntival, e a hemorragia resultante que pode ocorrer em alguns olhos. Irritação no pós-operatório por suturas expostas também podem ser eliminada. Além disso, o tempo cirúrgico e inflamação ocular são reduzidos devido à não realização dos passos anteriormente descritos, resultando numa recuperação visual mais rápida. ${ }^{(15)}$ Entre as possíveis desvantagens relacionadas à vitrectomia 25-gauge comparada, por exemplo, a 20-gauge seria a maior dificuldade em realizar a vitrectomia do vítreo periférico devido a maior flexibilidade dos instrumentais utilizados na 25gauge que resultaria numa pior movimentação do globo ocular no intraoperatório. ${ }^{(8,16)}$ Dessa forma, um maior vítreo periférico residual ocasionaria maior área de tração retiniana e consequentemente maior risco de rasgaduras e descolamentos da retina. No presente trabalho, as cirurgias foram realizadas com a 25 -gauge por se tratar em sua totalidade de cirurgias de baixa complexidade e com excelente potencial visual final.

Teoricamente, complicações potenciais inerentes a vitrectomia podem ocorrer em ambos os tipos de vitrectomia, como descolamento de retina, hipotonia ocular, membrana epirretiniana, endoftalmite e hemorragia expulsiva. Em nossa série, a única complicação observada após 3 meses de seguimento foi a presença de um edema macular cistóide (EMC), tratado clinicamente.

Não existem estudos clínicos comparando o desempenho visual, sensibilidade ao contraste, e qualidade de vida entre pacientes pseudofácicos com LIO monofocal e multifocal que apresentam queixas significativas de moscas volantes. Não sabemos se os pacientes com implante de lentes intraoculares (LIOs) multifocais têm menor tolerância a opacidades vítreas e floaters do que os pacientes com implante de LIOs monofocais. Estudos prospectivos serão necessários para esclarecer esses fatos.
A propriedade de difração da LIO multifocal foi primeiramente descrita por Fresnel, em 1818, um fenômeno que ocorre com as ondas quando elas passam por um orifício ou contornam um objeto cuja dimensão é da mesma ordem de grandeza que o seu comprimento de onda. ${ }^{(5)}$ A difração é o nome dado historicamente à transição oscilatória entre a luz e a sombra quando a luz é obstruída por um anteparo. Quando temos um anteparo com uma abertura, se verificado em detalhe a região de transição entre a luz e a sombra, é possível observar a presença de oscilações de diferentes intensidades próximas à região da borda. Posteriormente, estes fatos forma comprovados experimentalmente e chamou-se de ponto de Arago que ajudou a confirmar a importância das zonas de Fresnel. ${ }^{(17-18)}$ Isto poderia explicar porque pacientes queixam-se quando uma opacidade vítrea passa pelo seu campo de visão, e porque a vitrectomia ajuda na melhora dos sintomas e na qualidade visual, especialmente para perto.

Concluindo, nosso estudo mostrou que em pacientes cuidadosamente selecionados a remoção das opacidades vítreas (floaters) usando a vitrectomia transconjuntival 25-gauge se mostrou segura, e resultou em alto nível de satisfação dos pacientes, com resolução dos sintomas na sua quase totalidade, e uma melhora subjetiva da qualidade funcional da visão.

\section{ResUmo}

Objetivo: O objetivo do presente estudo é relatar o resultado visual obtido após realização de vitrectomia via pars plana 25 gauge (VVPP-25-gauge) em pacientes com opacidades vítreas persistentes e debilitantes previamente submetidos à cirurgia de facectomia com implante de lente intraocular (LIO) multifocal. Métodos: Estudo prospectivo de 14 olhos com implante de LIO multifocal e presença de opacidade vítrea submetidos à VVPP-25-gauge. Foram avaliados acuidade visual sem correção (AVSC), acuidade visual com correção (AVCC) para longe, acuidade visual para perto corrigida para longe (AVPC) e equivalente esférico (EE) antes e 3 meses pós-vitrectomia. Um questionário de satisfação e melhora visual foi administrado antes e após a realização da vitrectomia para avaliar a satisfação dos pacientes. Resultado:Após três meses da VVPP25-gauge, a média das acuidades (logMAR) era: AVSC, $0,10 \pm 0,14 ; A V C C, 0,02 \pm 0,08 ; A V P C, 0,00 \pm 0,0 ; E E,+0,16$ $\pm 0,34$. Treze olhos (92,9\%) apresentavam AVSC de 20/40 ou melhor, e 11 (78,5\%) de 20/25 ou melhor. Dez (90,9\%) pacientes demonstraram satisfação com o procedimento cirúrgico, obtendo eliminação de todos os floaters em 13 olhos $(92,8 \%)$. Apenas um paciente apresentou insatisfação com o resultado visual final devido ao aparecimento de edema macular cistóide pós-vitrectomia. Conclusão: $A$ 
remoção das opacidades vítreas, usando a VVPP-25-gauge se mostrou segura, e resultou em alto nível de satisfação dos pacientes, com resolução dos sintomas na sua quase totalidade, e uma melhora subjetiva da qualidade funcional da visão.

Descritores: Vitrectomia; Implante de lente intraocular; Acuidade visual; Extração de catarata; Satisfação do paciente; Resultado de tratamento.

\section{REFERÊNCIAS}

1. Coffee RE, Westfall AC, Davis GH, Mieler WF, Holz ER. Symptomatic posterior vitreous detachment and the incidence of delayed retinal breaks: case series and meta-analysis. Am J Ophthalmol 2007; 144: 409-413.

2. Parnes RE, Zakov ZN, Novak MA, Rice TA. Vitrectomy in patients with decrease visual acuity secondary to asteroid hyalosis. Am J Ophthalmol 1998; 125: 703-704.

3. Mossa F, Delaney YM, Rosen PH, Rahman R. Floaterectomy: combined phacoemulsification and deep anterior vitrectomy. J Cataract Refract Surg 2002; 28: 589-592.

4. Tsai W-F, Kenyon KR, Takahashi M, et al. Treatment of vitreous floaters with neodymium Yag laser. Br J Ophthalmol 1993; 77: 485-488.

5. Quintyn JC; Brasseur G. Vitrectomy for floaters. J Fr Ophthalmol 2004; 27(5):491-5.

6. Hoerauf H, Müller M, Laqua H. Vitreous body floaters and vitrectomy with full visual acuity. Ophthalmologe 2003; 100(8): 639-43

7. Schiff WM, Chang S, Mandava N, Barile GR. Pars plana vitrectomy for persistent, visually significant vitreous opacities. Retina 2000; 20(6): 591-596.

8. Lakhanpal RR, Humayun MS, Juan E, et al. Outcomes of 140 consecutive cases of 25-gauge transconjuntival surgery for posterior segment disease. Ophthalmology 2005; 112( 5): 817-824

9. Romero P, Salvat M, Almena M, Baget M, Méndez I. Experience with 25 -gauge transconjunctival vitrectomy compared to a 20-gauge system. Analysis of 132 cases. J Fr Ophthalmol. 2006; 29(9): 1025-32.
10. Murakami K, Jalkh AE, Avila MP, Trempe CL, Schepens CL. Vitreous floaters. Ophthalmology 1983; 90: 1271-1276.

11. Kakehashi A, Inoda S, Shimizu Y, Makino S, Shimizu H. Predictive value of floaters in the diagnosis of posterior vitreous detachment. Am J Ophthalmol 1998; 125: 113-115.

12. Tanner V, Harle D, Tan J, Foote B, Williamson TH, Chignell AH. Acute posterior vitreous detachment: the predictive value of vitreous pigment and symptomatology. Br J Ophthalmol 2000; 84: 1264-1268.

13. Bonner RF, Meyers SM, Gaasterland DE. Threshold for retinal damage associated with the use of high-power neodymium-YAG lasers in the vitreous. Am J Ophthalmol 1983; 96: 153-159.

14. Brown GC, Scimeca G, Shields JA. Effects of the pulsed neodymium: YAG laser on the posterior segment. Ophthalmic Surg 1986; 17: 470-472.

15. Ripandelli G; Coppé AM, Parisi V, Olzi D, Scassa C, Chiaravalloti A, Stirpe M. Posterior vitreous detachment and retinal detachment after cataract surgery. Ophthalmology 2007; 114: 692-697.

16. Ibarra MS, Hermel M, Prenner JL, Hassan TS. Longer-term outcomes of transconjunctival sutureless 25 -gauge vitrectomy. Am J Ophthalmol 2005; 139: 831-836.

17. Davison JA, Simpson MJ. History and development of the apodized diffractive intraocular lens. J Cataract Refract Surg 2006; 32:849-858.

18. Born M, Wolf E. Principles of Optics; Electromagnetic Theory of Propagation, Interference, and Diffraction of Light, 5th ed. New York, NY, Pergamon Press, 1975.

\section{Endereço para correspondência: \\ Patrick F. M. Tzelikis \\ SQN 203, bloco G, apto 405 \\ CEP 70833-070 - Brasília (DF), Brasil \\ E-mail: tzelikis@terra.com.br}

